\title{
Character Pleasantness in Malaysian Animated Cartoon Characters
}

\author{
Mohd Rosli Arshad $^{1 *}$, Kim Hae Yoon ${ }^{2}$ and Ahmad Azaini Manaf ${ }^{3}$ \\ ${ }^{1}$ Graduate School of Design, Dongseo University, Busan, South Korea \\ ${ }^{2}$ Graduate School of Design, Dongseo University, Busan, South Korea \\ ${ }^{3}$ Faculty of Applied and Creative Arts, University Malaysia Sarawak. Malaysia
}

\begin{abstract}
Current global trends have proved the creative industry to be one of the important sources of economic growth among developed countries. Creativity and its importance for Malaysia have made it imperative for any business organization to use creativity in a range of ways including multimedia content and animation. Malaysian animation viewers are rapidly influenced by digital media entertainment. The rise of such entertainment tends to drive them away from understanding what lies behind it that affect their emotion and thoughts. Therefore, the focus of this paper is to look into the experiences of "pleasantness" in viewer's emotions that stimulate the perception of pleasure when watching Malaysian animated cartoon characters. A descriptive and One-Way Anova will be implemented in this study to examine the design aesthetics and perception from the animation viewers that affects the psychological experiences in emotions that determines the pleasantness feeling. Overall, the results indicate that perceived pleasantness on Malaysian animated cartoon characters did not differ between age and gender. We believe this finding will benefit the creative content creators and help them to understand more about local animation viewers.
\end{abstract}

\section{Introduction}

Animation films, as a part of entertainment has long been recognized and accepted as a media content that influences people in any society. The importance of character design in animation and other media entertainment may have an indirect impact on the emotion of the viewers. According to Hodges's 2011 (as cited in Adi Bunyamin, Dahlan Ghani \& Amir Asrieff Amir Hussin, 2017), the importance of graphically rendered characters has been paramount in this new form of entertainment. He suggested that a successful animated character is comparable to a successful actor whose popularity can be used to sell products, concepts, information etc. A simple graphic representation becomes instantly recognizable, appealing, emotionally accessible and engaging. Animated feature films are extremely expensive to create, costing some studios hundreds of millions of dollars. On a large scale

\footnotetext{
*Corresponding author: mrosli@unikl.edu.my
} 
production, it is critical that every detail is perfect. In order to avoid failure, it is important to understand human visual attention when designing characters of animated films.

In the Malaysian animation industry, we have seen many products failed in the market. One prime example is the big budget local animated feature film called $\mathrm{Oh} \mathrm{La} \mathrm{La} \mathrm{in} \mathrm{2015,}$ that spent almost RM 4 million and has failed to attract local audiences. Even though it is considered as the first Malaysian musical animated film, it failed miserably. Aesthetically, the entire film was visually not up to industry standards and one of the reasons behind it is the lack of quality in the design aesthetics. However, there are also other animated feature films which are very successful in the local market. For examples, films such as Upin and Ipin (2007), Boboiboy (2011) and Ejen Ali (2016) have successfully implemented the aesthetic of designs that helped to flourish Malaysian animation visual contents. Thus, this study explores the phenomenological experiences of "pleasantness" in viewers' emotion that stimulate the sensory perception of feeling pleasure and excitement when watching Malaysian animated cartoon characters in terms of design aesthetics. The aim is merely to understand the difference between gender and age that influence the sense of pleasure on character design aesthetics in animation film and to identify whether gender and age preferences gave a significant impact to the overall animation films.

\section{Research background}

\subsection{The Malaysian animated films}

Malaysian animated film is maturing and its contents has been accepted globally. The progress of technology and multimedia has tremendously assisted in the development of animation contents in Malaysia. It all started with the initiative of Kamn Ismail in 1995 where he produced the first animated local series called Usop Santorian. Since then, the demand of local animation kept increasing due to the effort taken by Multimedia Digital Economy Corporation $(\mathrm{MDEC})^{1}$ to boost and promote local animation to the international market. Over time, Malaysian animation products have successfully attracted many audiences not just locally but globally as well. In the beginning, local animation content was created based on Malaysian culture as well as to promote harmony among the different society in Malaysia (Mohd Nazri \& Hassan, 2013). Later, the Malaysian animation content began to change to a more universal content in order to attract international viewers. Bola Kampung (2006) animated series was a starting point for the local animation titles to have been accepted globally. The series became the first local animation to break into the international market broadcast network. In November 2007, it aired in 16 Asian countries over the Disney Channel (Hassan, 2016, p.153). It was then followed by Upin and Ipin (2007), which became a huge phenomenon especially in South East Asian countries that shared similar cultures and language. The popularity of Upin and Ipin has lead the commercial success of Malaysia's first CGI-animated film, Geng: The Adventure Begin (2009), which features Upin and Ipin that successfully earned RM 6.31 million and being considered as one of the most successful computer 3D animated television series in Malaysia (Abdul Ghani, 2015). As a result, Upin and Ipin has opened a way, to many other local animation content such as Super Tots (2010), Boboiboy (2011), Dunia Eicak (2012) and recently Ejen Ali (2016).

${ }^{1}$ Malaysia Digital Economy Corporation (MDEC) is an agency under Ministry of Communication and Multimedia Malaysia that responsible to build, organizing and promoting digital economy and communications technology (ICT) in Malaysia. For more information, please refer to www.mdec.my. 


\subsection{Malaysian animated cartoon characters}

Anime (Japanese manga comic characters) has very large following in Malaysia. The popularity of Japanese animation has influenced the local designers and animators. In early 1990 's where local animation industries is just started, local animation studios had brought in the anime-style to the productions they worked on. This is because early animators had no proper training in art and design nor were exposed to the various styles of animation of other countries (Hassan, 2016). As time goes by, many local animators started to realize the importance of design aesthetics in their creative works and started to explore their own root.

Today, more local animation company has developed their own unique animation stories and appealing animation characters. For animators, when designing the animation characters, they have almost the same art direction. Visual style, color and demographic factors has to be considered before coming up with a finishing design for the characters. From Usop Sontorian animation in 1996 until today, the Malaysian cartoon characters have changed drastically from anime-like design into local influences visual style designs that capture the imaginations of animation enthusiasts and are widely appreciated by Malaysian animation viewers.

\subsection{Design aesthetics in animated films characters}

The importance of design and color in animated films is very significant in order to portray a specific mood or emotion. Sajjad (2012) stated that the combination of basic shapes and colors are relied heavily on character design. The artist with the intention to create a greater emotional response in the viewer emotion uses the appropriate design and color. Appealing designs contribute to a sense of emotional engagement between the visuals and the ultimate goal depicted to the audience. The great character designer visualizes inspiration from history, culture and everyday life. Culture plays a significant role in defining a good and aesthetic design. According to Welsh-Asante (1994), an aesthetic draws upon the history, mythology, motif and creative ethos of a group of people in which is something obtained by fully understanding the ways of the certain community. By definition, character means the mental and moral qualities distinctive to an individual ("character"). It is also to determine how the person looks, acts, speaks, and even interacts with others. Su and Zhao (2011) defines it as the "design of human or humanlike characters of distinctive uniqueness and rich features for all kinds of visual media" (p.12). The perfect combination of colors in design creates a wealth of information that supports the idea that graphics enhance the emotional experience. Ionescu, Lambert, Coquin, \& Buzuloiu (2007) put forward the claim that color is a major feature of animation movies: almost each movie uses a particular color palette, which is related to its artistic content, and the colors are selected and mixed by the artist according to art concepts to express particular feelings or to induce particular sensations in emotions. In fact, in animation the aesthetics of design and colors is not only applicable for the characters but is also essential to the visual background and animation environment to establish the mood or scenario of a scene that will integrate with the characters involved.

It is apparent from the previous studies that design and color aesthetic plays an important role in revealing human emotion towards animation contents. Recent evidence suggests that online gaming contents positively give hedonic or pleasantness experience towards the players and it significantly affects all dimensions of emotions according to PAD Model developed by Mehrabian and Russell in 1974 (Huang, Ali, \& Liao, 2017). Online gaming is almost similar representation of animated films where all the visual is created using the same process where the design of characters and environment is developed at early stage. Thus, the characteristics of design and color aesthetics is significantly important and serve a 
promising effect to the viewer's emotion due to the fact that the content can be created as imaginatively as possible (Napier,2001, p.157).

\subsection{Pleasantness (emotion)}

Influence on emotion is a key factor to satisfy the movie viewers. One way to understand emotion is through positive and negative feeling. In theory, the psychology effects when people are asked the emotional factors of watching film, the common sense answer is to be entertained, to escape boredom, to get respite from their problems, to get new experience or information and, sometimes, to get an artistic thrill (Tan, 1996, pp. 17-18). From this finding, we can see that human beings are looking for relief, comfort or escape in their lives and watching movies is one way to reconnect or reawaken the sense of pleasure that effects their emotion and cognitive abilities.

Emotion knowledge is recognized as being split into two or three-dimensional structure, with positive-negative valence, activity or arousal, and potency or dominance (Dormann, 2006). The dimension of pleasantness is defined as the positive-negative valence of emotion and ranges from unpleasant to pleasant (Clore, 1994). Many psychology researchers have demonstrated that induced pleasure encourages the viewer to explore the stimuli in more detail. For example, Isen, Daubman, \& Nowicki, (1987) purported that positive emotions increase an individual's sensibility to the stimuli in an environment. The word pleasantness is referring to pleasure or happiness. By the term positive effect, it means a conscious feeling of pleasure, a quintessentially subjective phenomenon. Conscious pleasure is the only form of pleasure of which many people can conceive (Berridge, 2003). Mehrabian and Russell (1974) developed environmental psychology model called PAD (Pleasure, Arousal and Dominance) or emotional state model to describe human psychology that conceived three basic dimensions of emotion responses that indicate people's state of feeling. They suggested that pleasure as a continuum range from extreme pain or unhappiness to extreme happiness and used adjective such as happy-unhappy, pleased-annoyed, and satisfied-unsatisfied to define a person's level of pleasure (Huang, Ali, \& Liao, 2017).

\section{Research methodology}

The groundwork of this paper is to explore the experience of pleasantness in animated cartoon characters among Malaysian animation viewers. In order to investigate whether the character design aesthetics contribute to people's perception and emotion, we hypothesized that there is a significant difference between gender and age among the respondents. A set of questionnaire were used which consists of 9 items with categorical and five-point Likert scale sections ranging from 1 (Strongly Disagree) to 5 (Strongly Agree). Statistical analysis was performed in order to examine the difference between characteristics in emotion and design aesthetics in cartoon characters. A questionnaire's survey has been conducted to $N=143$ respondents consists of Malaysian youth age from 17 to 27 years old. The questionnaire employed in this research contained four parts, which in the first part is related to the demographic variables of the respondents such as gender and age. The second part contains nine selected titles of Malaysian animated films with the character lineup to give an emotional responsiveness to the respondents. These animation films are Upin and Ipin (2007), Bola Kampung (2007), Super Tots (2010), Boboiboy (2011), Dunia Eicak (2012), The Amazing Awang Kehnit (2014), Puteri (2014), Rimba Racer (2015) and Ejen Ali (2016). The last part was divided into three groups of questions that asked respondents' awareness and perception 
about local animated characters, character design aesthetics and psychological effects towards pleasantness in animated cartoon characters. In order to estimate the result, first we will use a frequency table for three items regarding the respondent awareness and perception about selected case studies for this research purposes.

Second, the Analysis of Variance (One-way ANOVA on rank) is used to determine the significance between independent variables groups in this paper. The rank statistical tests, is conducted to examine two categories variables, which is design aesthetics and feeling pleasantness between respondent's gender and age.

\section{Research analysis and results}

The goal of the current study was to examine the design aesthetics and perception from the animation viewers that affects the psychological experiences in encouraging positive emotions that determine the pleasantness feeling. The data in Table 1 were analyzed in frequency table to show the differences between gender and age to obtain numbers of participation in this research. Likewise, descriptive statistics concerning like to watch and like the appearance is presented in table 2 .

Table 1: By frequency $(\mathrm{N}=143)$ for Gender and Age

\begin{tabular}{c|c|c|c}
\hline \multicolumn{2}{|c|}{ Gender } & \multicolumn{2}{c}{ Age } \\
\hline & Frequency \& $(\%)$ & & Frequency \& $(\%)$ \\
\hline Male & $78(54.5 \%)$ & less than 20 & $34(23.8 \%)$ \\
Female & $65(45.5 \%)$ & $20-23$ & $43(30.1 \%)$ \\
Total & $143(100 \%)$ & $24-27$ & $66(46.2 \%)$ \\
& & Total & $143(100 \%)$ \\
\hline
\end{tabular}

Table 2: By frequency $(\mathrm{N}=143)$ for Awareness and Perception

\begin{tabular}{c|c|c|c}
\hline \multicolumn{2}{c|}{ Like to watch (Awareness) } & \multicolumn{2}{c}{ Like the appearance (Perception) } \\
\hline & Frequency \& (\%) & Frequency \& (\%) \\
\hline Yes & $94(65.7 \%)$ & Yes & $92(64.3 \%)$ \\
No & $49(34.5 \%)$ & No & $51(35.7 \%)$ \\
Total & $143(100 \%)$ & Total & $143(100 \%)$ \\
\hline \multicolumn{2}{c|}{ Factor's why I like the characters (Perception) } & Frequency \& (\%) \\
\hline \multicolumn{3}{c}{ Character's role } & $29(20.3 \%)$ \\
\multicolumn{3}{c}{ Character's appearance } & $32(22.4 \%)$ \\
Character's dialogue & $18(12.6 \%)$ \\
Character's theme & $57(39.9 \%)$ \\
None & $7(4.9 \%)$ \\
& Total & $143(100 \%)$ \\
\hline
\end{tabular}

Table 1, indicates the total sample of gender is $\mathrm{N}=143(\mathrm{M}=1.45, \mathrm{SD}=0.50)$ Malaysian youth consists of male is $54.5 \%(n=78)$ compared to female $45.5 \%(n=65)$. Next table, summarizes the respondents aged $(\mathrm{M}=2.22, \mathrm{SD}=0.80)$ less than 20 years old accounted for $23.8 \%(n=34), 20-23$ years old is $30.1 \%(n=43)$ and $24-27$ years old for about $46.2 \%(n=66)$ which brings the highest participation in this study.

In Table 2, the percentage of awareness among sample shows that like to watch local animation $(M=1.34, S D=0.47)$ indicates that $65.7 \%(n=94)$ of the total respondents really love to watch Malaysian animation compared to $34.3 \%(n=49)$ dislike and preferred to like 
non-local or foreign animation. In general, the results clearly show that most of the respondents who took part in this survey really love to watch local animation. Obviously, it will benefit the purposes of this study and might influence on the results of this study. The findings from Table 2 also indicate that the perception of like the character appearance in local animation $(\mathrm{M}=1.36, \mathrm{SD}=0.48)$ have showed the percentage of $64.3 \%(\mathrm{n}=92)$ from the respondents which give an impression that the character's appearance gave a significance impact into their emotion. Further analysis is to identify the main factor that contributes to their personal preferences of why they like the characters in the animation $(M=2.87$, $\mathrm{SD}=1.27$ ). The data revealed that character's theme shows the highest percentage $39.9 \%$ $(\mathrm{n}=57)$ compared to others. While data from character's appearance and character's role appears to have a $22.4 \%(n=32)$ and $20.3 \%(n=29)$ that have not so much different percentage which strongly suggested that character appearance and its role indeed play an important factor in animation films. Besides, the results can be connected back with the like the character appearance results which majority of the respondents strongly believe that characters in local animation have the positive psychological effect into their emotion. The relationship between character's theme and character's appearance is very important in the early stage of the animation production. The theme and concept of the animation are carefully planned to ensure the visual looks of the final products is according to the storyline.

In this test, the exact significance level was generated and we use the exact $p<.05$ to determine our results, and not to reject the null hypothesis of the p-value if greater than 0.5 (i.e., $\mathrm{p}$ >.05). A Mann-Whitney test was conducted to determine if there are any significant differences in character design aesthetics scores between males and females. Mean scores result reveals that males (mean rank $=73.35$ ) and females (mean rank $=70.38$ ) were not statistically significantly different, $U=2.430, z=-.4 .55, p=.649(p>.05)$. Age scores was conducted in Kruskal-Wallis $\mathrm{H}$ test reveals that distribution scores were dissimilar for all groups range from less than 20 years old to 27 years old. Median scores were not statistically significantly different between age groups, $X^{2}(2)=4.007, p=.135(p>.05)$. While for the role of colors also brings a non-significant results, $\mathrm{X}^{2}(2)=1.666, \mathrm{p}=.435(\mathrm{p}>.05)$. As for the pleasantness feeling, the distribution of mean as assessed by visual inspection of frequency, indicates that gender distributions were not similar between males (mean rank = 73.83 ) and females (mean rank =69.80), $\mathrm{U}=2.392, \mathrm{z}=-.619, \mathrm{p}=.536(\mathrm{p}>.05)$ and were not significantly different. Finally, test for age groups showed that the distribution of visual inspection in boxplot were highly dissimilar for all age groups. Median scores for age groups and pleasantness were not statistically significant where $X^{2}(2)=4.351, p=.114(p>.05)$. These results strongly suggest to keep the null hypothesis and shows that there is no significant difference between gender and age groups when comparing with pleasantness feeling towards character designs aesthetics in local animated cartoon. Based on our observations from the results above, we found that the character design plays an important role in determining the successful factor in local animation film. We had studied $\mathrm{N}=143$ from the population consists of Malaysian youth age between 17 to 27 years old. We conclude that there is no difference between males and females when associating the feeling of pleasantness and character design aesthetics. However, the findings can be argued because there are certain conditions that can be questioned. The possible explanation for this might be:

i. Focusing on local animation products only, might affect the overall feeling of the respondents.

ii. The selected animation characters from the case studies might not interest them.

iii. The respondents might be confused and find it difficult to distinguish the aesthetics of each characters because the design is different. 
Nevertheless, we found that the results have shown a strong evidence that the character animated cartoon in Malaysian animation film have successfully given a significant impact to the emotion of the animation viewers.

\section{Research finding and discussions}

The aim of the present study is to examine how the participants perceived pleasantness that may affect their feelings when watching Malaysian animated cartoons. The strength of this paper is to highlight the importance of understanding human emotion towards local animated cartoon characters. By examining the feeling of "pleasantness", we tried to understand what lies behind their feelings that stimulate the sensational feeling of pleasure when watching local animated cartoon characters. However, some weaknesses could be identify in this study. The clearest point of view in this paper is only to discuss the findings on the positive valence of emotion that described the conscious pleasure of watching animated characters but not the negative valence that leads to the feeling of unhappiness and annoyance. The PAD (Pleasure, Arousal and Dominance) psychology model is not fully utilized to examine the emotional response since the model conceived three basic dimension. Here, we only looked at one dimension, which is 'Pleasure' as a basic dimension to comprehend the meaning of pleasantness to describe level of pleasure of human emotion.

At the beginning, we hypothesized that there are higher chances of differences between genders and age. The results indicated that there were no significant differences between gender and age when pleasantness is perceived towards animated cartoon characters. As mentioned in the literature review, character appearance in animation was considered as one of the important element in animation. The results of this study has shown a positive result which supports previous research. Another important finding is the character's theme in the story is the main factor that contributes to the sense of pleasantness in animation. This outcome reveals that the theme for the overall looks of the animation including the character role and theme in the animation gave a significant impact to the emotional states that gives pleasurable experience to the viewers. Creating a dominant and attractive character design in animation is one of the key features to enhance the mood of the animation viewers.

This study may be important for the exploration of relations between emotion and the animation content. By defining the importance of understanding animation viewers, it symbolizes an extended meaning to the animation makers and the animation industries. From the business and marketing perspective, the results of this study provide significant insight for the purposes of producing and selling animation products in Malaysia. According to Hassan (2000), animation production must be viewed as a business that promotes folklore heroes and stories to the local audience. The local society shall become the consumer of local culture. In fact, one of the advantages of having a good character design is the translation into successful IP (Intellectual Property). The important aspect of creative work is ownership of the intellectual property. As an advantage, popular cartoon character has the value to position itself in promoting local brands. Hence, despite the limits, the data from the present study appear important for research concerning the relationship between animation viewer's emotion and the animation contents. It is beneficial for the creative content creators who produce animation for the Malaysian viewers. 


\section{Conclusion}

Therefore, we hope that this study will benefit the creative content creators to understand how Malaysian animation viewers perceived pleasantness. This is to develop successful commercialized animation content, especially on the creation of animated characters. Finally, we would like to highlight the results that suggest the character's theme is the main factor that contributed to the psychological effects to the viewers and may be a worthwhile direction for future work. We recommend that further study should explore the perception and emotion that stimulate the sense of enjoyment among animation viewers especially local audiences. Additional studies need to be done to understand the psychological and phenomenological experiences from the eyes of the local animation viewers.

\section{Acknowledgement}

This paper is part of author's doctoral research sponsored by the Majlis Amanah Rakyat (MARA) and supported by the Universiti Kuala Lumpur, Malaysia.

\section{References}

Adi Bunyamin, Dahlan Ghani, Amir Asrieff Amir Hussin, M. Z. K. (2017). Technological advancement through character design: Darth vader as case study. Annales Series Historia et Sociologia, 1, 1-8. https://doi.org/10.19233/ASHS.2017.01

Abdul Ghani, D. Bin. (2015). Upin \& Ipin: Promoting Malaysian culture values through animation. Historia Y Comunicacion Social, 20(1), 241-258. https://doi.org/10.5209/revHICS.2015.v20.n1.49558

Berridge, K. C. (2003). Pleasures of the brain. Brain and Cognition, 52(1), 106-128. https://doi.org/10.1016/S0278-2626(03)00014-9

Ionescu, B., Lambert, P., Coquin, D., \& Buzuloiu, V. (2007). Color-based content retrieval of animation movies: A study. CBMI'2007 - 2007 International Workshop on Content-Based Multimedia Indexing, Proceedings, 295-302. https://doi.org/10.1109/CBMI.2007.385425

Isen, A. M., Daubman, K. A., \& Nowicki, G. P. (1987). Positive affect facilitates creative problem solving. Journal of Personality and Social Psychology, 52(6), 1122-1131. https://doi.org/10.1037/0022-3514.52.6.1122

Hassan Abd. Muthalib. (2000). Sang Kancil - Ke Mana Kau? Filem animasi di era globalisasi - masa depan dan cabaran. Working paper presented in November 2000 at Minggu Sastera Kebangsaan, Dewan Bahasa dan Pustaka, Kuala Lumpur.

Hassan Abd. Mutalib. (2016). From mouse deer to mouse: 70 years of Malaysian animation ( $1^{\text {st }}$ edition), Kuala Lumpur: Akademi Seni Budaya dan Warisan Kebangsaan (ASWARA).

Huang, M., Ali, R., \& Liao, J. (2017). The effect of user experience in online games on word of mouth: A pleasure-arousal-dominance (PAD) model perspective. Computers in Human Behavior, 75, 329-338. https://doi.org/10.1016/j.chb.2017.05.015

Napier, S.J. (2001) Anime from Akira to Princess Monokoke: Experiencing Contemporary Japanese Animation ( $1^{\text {st }}$ Ed). New York: Palgrave Macmillan. 
Sajjad, S., Mohsin, S., Riaz, S. \& A. Abdullah. (2012): Enemy Character Design for Graphical Imagery Therapy (GIT) Game. International Journal of Information and Education Technology, 2, 4 (August 2012), 345-347.

Tan, E. S. (1996). Emotion and the structure of narrative film. Mahwah, NJ: Lawrence Erlbaum Associates, Publishers. 